Supplement of Atmos. Meas. Tech., 11, 4707-4723, 2018

https://doi.org/10.5194/amt-11-4707-2018-supplement

(C) Author(s) 2018. This work is distributed under

the Creative Commons Attribution 4.0 License.

(c) (1)

\title{
Atmospheric Measurement Techniques
}

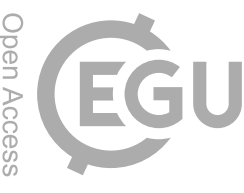

Supplement of

\section{Differences in ozone retrieval in MIPAS channels A and AB: a spectroscopic issue}

Norbert Glatthor et al.

Correspondence to: Norbert Glatthor (norbert.glatthor@kit.edu)

The copyright of individual parts of the supplement might differ from the CC BY 4.0 License. 
Manuscript prepared for Atmos. Meas. Tech. with version 2015/04/24 7.83 Copernicus papers of the $\mathrm{LT}_{\mathrm{E}} \mathrm{X}$ class copernicus.cls.

Date: 3 August 2018

\section{Comparison of spectral parameters of ozone lines in MIPAS pf3.2 and in HITRAN-2008}

In Figure 1 (top) the intensities of the ozone lines used for channel $\mathrm{A}$ and $\mathrm{AB}$ retrievals of the HITRAN-2008 database are plotted against the corresponding intensities of the MIPAS pf3.2 spectroscopy. The bottom panel shows the relative differences. Except of six outliers the line intensities are identical in the spectral region of the channel $\mathrm{AB}$ microwindows (cf. main paper). The line intensities in the channel A microwindows are also identical for the strongest lines with $\mathrm{S}>10^{-22}$ $\mathrm{cm}^{-1} /\left(\right.$ molecule $\left.\times \mathrm{cm}^{-2}\right)$ at $296 \mathrm{~K}$. The relative differences between the weaker lines are mostly below $3 \%$ and up to $\pm 7 \%$ for a few very weak lines only.

Figure 2 (top) shows the correlation between the air-broadened halfwidths $\gamma_{a i r, 0}$ of the ozone lines of the HITRAN-2008 database and the MIPAS pf3.2 spectroscopy used for channel A and AB retrievals. The corresponding relative differences are presented in the bottom panel. The halfwidths of the strongest lines $(\log \mathrm{S}>-21.5)$ in the channel A microwindows are identical. Thus there is not such a distinct bias in the halfwidths of strong lines like between HITRAN-2008 and GEISA-2015 (cf. main paper, Fig. 8a). However there are also significant differences between $-10 \%$ and $+20 \%$ for the halfwidths of the weaker lines. Mostly the HITRAN-2008 values are larger than those of the MIPAS pf3.2 spectroscopy. Since the ozone profiles are nearly identical for use of the MIPAS pf3.2 or HITRAN-2008 spectroscopy (cf. main paper, Fig. 5a), these differences obviously have no large impact on the retrieval results. The correlation between the halfwidths of the ozone lines in the channel $\mathrm{AB}$ microwindows appears rather similar. Again, the halfwidths of the stongest lines are identical, but the HITRAN-2008 halfwidths are up to $25 \%$ larger for medium-sized and weak lines.

Figure 3 shows the coefficients $n$ for calculation of temperature dependence of the air-broadened halfwidths (cf. main paper). While the value of these coefficients is constantly 0.76 in the MIPAS pf3.2 spectroscopy, they vary between 0.71 and 0.86 in HITRAN-2008. However, as shown in Section 7.2.2 of the main paper, the effect of variations in $n$ of this magnitude on the air-broadened halfwidths is small only.

Figure 4 shows that the positions of the ozone lines used for channel AB retrievals are identical in both spectroscopies. In channel A there are small differences, but again for medium-sized and weak lines only. 

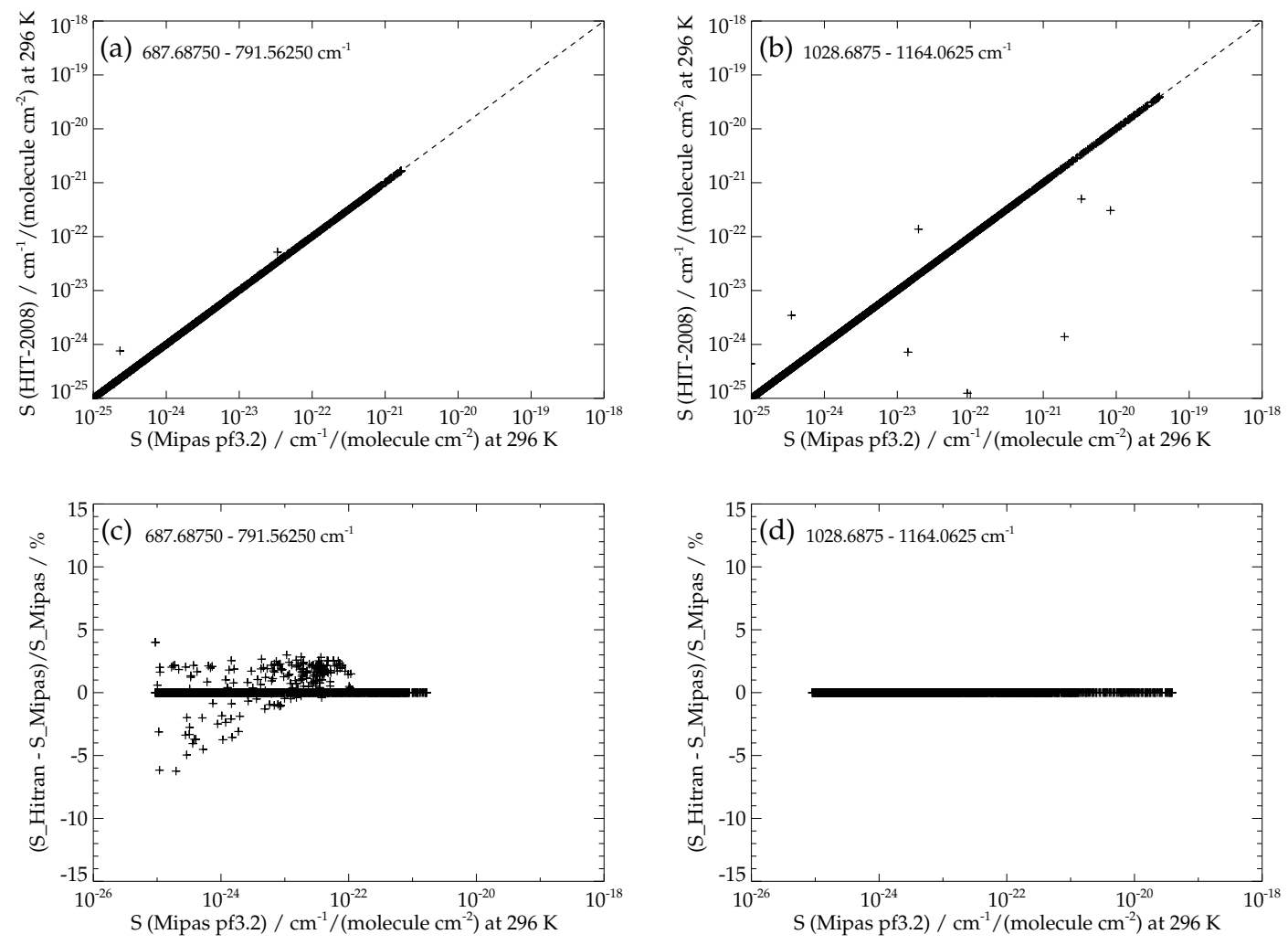

Figure 1. Ozone line intensities S of HITRAN-2008 versus MIPAS pf3.2 for the microwindows in MIPAS channel A (a) and AB (b), and relative differences for the microwindows in MIPAS channel A (c) and AB (d) . Note that the strong outliers, which can be seen in the top row, are not within the y-axis range of the difference plots. 

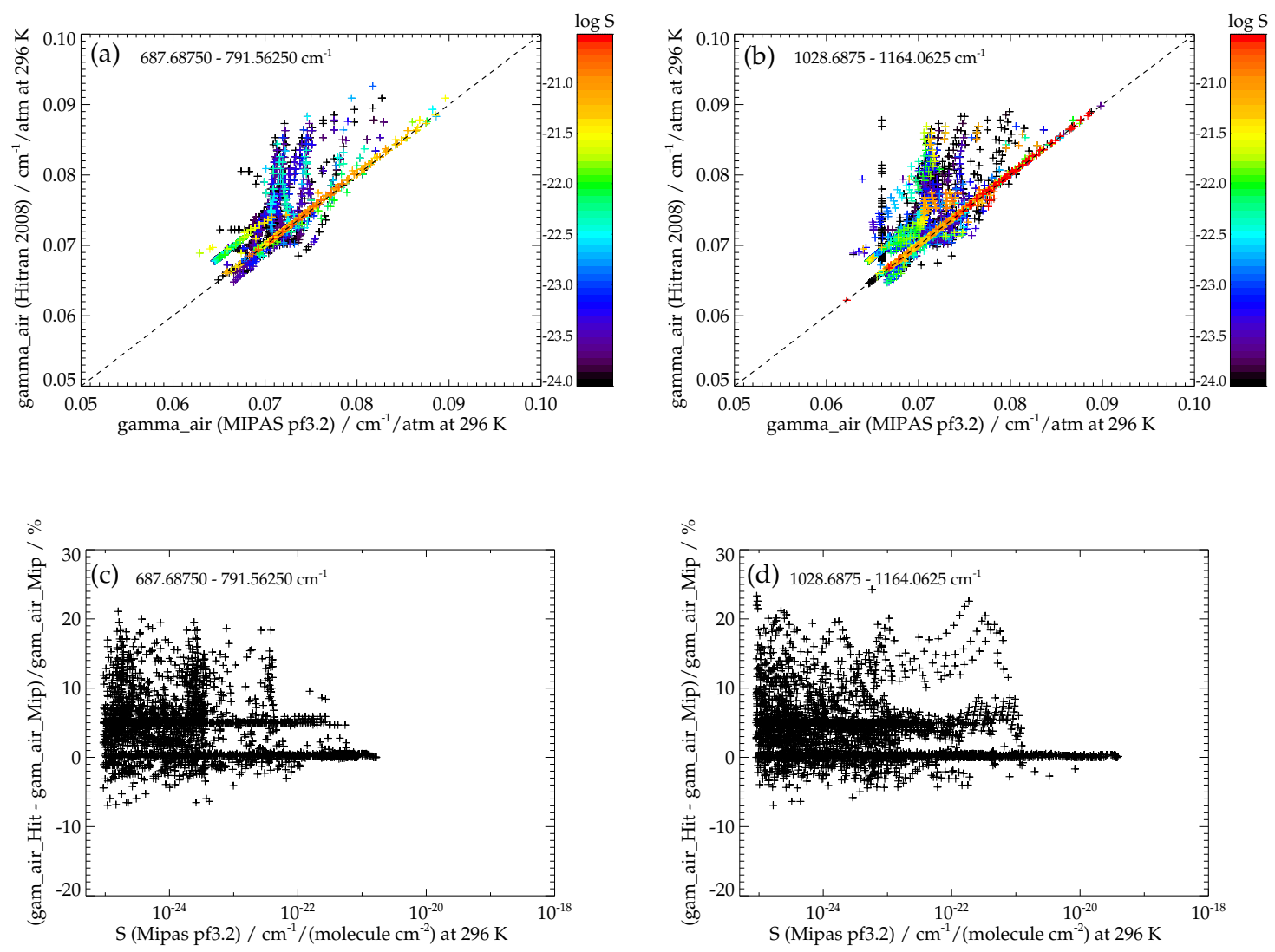

Figure 2. Air-broadened halfwidths $\gamma_{a i r, 0}$ of HITRAN-2008 versus MIPAS pf3.2 for the microwindows in MIPAS channel A (a) and $\mathrm{AB}$ (b), and corresponding relative differences (c, d). The halfwidths are colourcoded by the logarithm of the MIPAS pf3.2 line intensities S. 

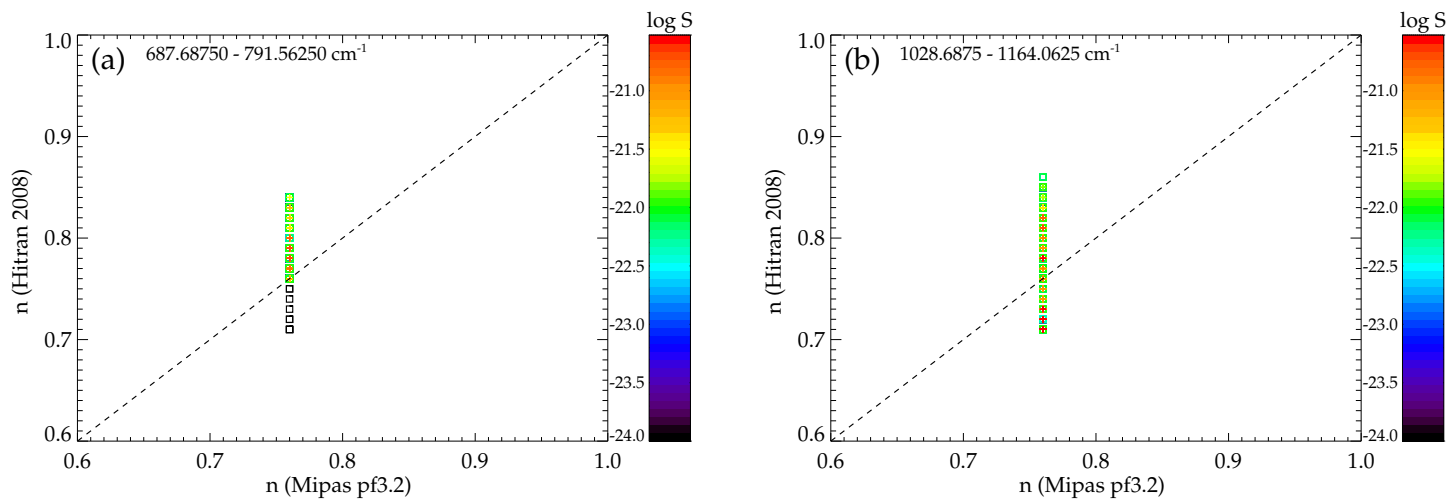

Figure 3. (a) Coefficients $n$ of temperature dependence of the air-broadened halfwidths of HITRAN-2008 versus MIPAS pf3.2 for the microwindows in MIPAS channel A. (b) Same as (a), but for the microwindows in channel AB. The coefficients are colour-coded by the logarithm of the MIPAS pf3.2 line intensities. Plotted squares/pluses denote coefficients associated to line strengths lower or larger than $1 \times 10^{-22} \mathrm{~cm}^{-1} /$ (molecule $\times \mathrm{cm}^{-2}$ ) at $296 \mathrm{~K}$.
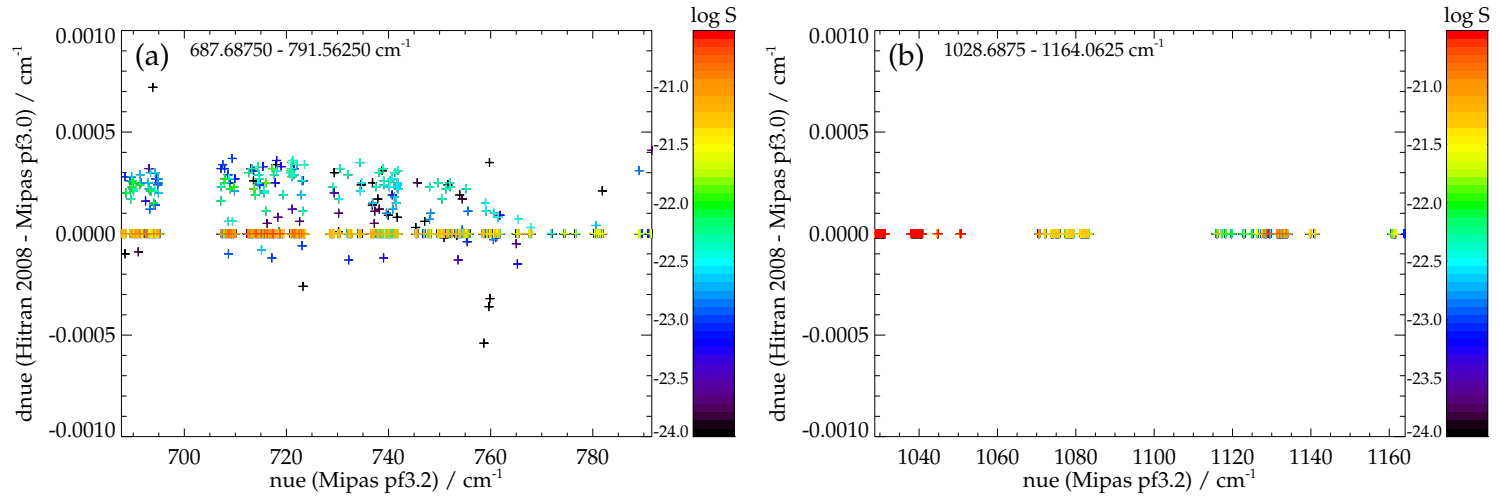

Figure 4. Relative differences dnue between ozone line positions in HITRAN-2008 and MIPAS pf3.2 for the microwindows in MIPAS channel A (a) and AB (b). 